\title{
Comics, Adoptionen oder doch das Original? - Goethes Faust im kompetenzorientierten Deutschunterricht
}

\section{Faust-Comics, Faust- children-books or Goethe's masterpiece Faust in German-literature-lessons}

\begin{abstract}
Goethe's Faust was long defined in Germany as an established part of literature lessons and as a part of canon, but a paradigm change in didactics and in literature science has challenged both. This essay takes a brief look at history of German-literature-lessons and at different methods of teaching and reading literature, especially Goethe's Faust - analytical, productive and creative. It gives a survey about children's books to Faust, comics and summaries and demonstrates the correlation between knowledge and reading comprehension.
\end{abstract}

KEYWORDS: Goethe; comics; children literature; history of German- literature-lessons; world literature; reading comprehension; methods; cross cultural reading.

\section{STATT EINER EINLEITUNG}

Goethe gehört zu den Klassikern der Weltliteratur, seine Werke werden als ein Kulturphänomen betrachtet und genießen kanonischen Stellenwert. Die Zuschreibungen Genie, Dichterfürst, Weltbürger, Großklassiker, nationale Stiftungslegende, Nationaldichter, herausragend, einmalig, brillant, fulminant, grandios, phänomenal, exzeptionell und andere ähnliche Superlative sind in Verbindung mit seinem Namen konstante Begleiter. Seine Werke sind in viele Sprachen übersetzt und werden rund um die Welt gelesen. Sie sind und werden weiterhin zusammengefasst, verfilmt, theatralisch 
aufgeführt, parodiert, persifliert und sind als Neuerscheinungen, Nacherzählungen und Überarbeitungen auch für Kinder und Jugendliche, aber auch als Comics, Manga und Hörbücher, Adoptionen einzelner Motive, Figuren, Textstellen, als Applikationen (Link, Link-Heer 1980) vorzufinden. Goethes Werke gelesen zu haben war einst das distinktive Merkmal und das Synonym für die Bildung schlechthin. Er war, ist und bleibt somit eine zentrale Figur im öffentlichen und zentralen (Bildungs)diskurs, wenngleich David Damrosch (2003) darauf hinweist, dass seine Werke nicht länger dem weltliterarischen Kanon angehören. Es werden Goethe-Ausstellungen initiiert, Vorlesungen auch für breite Öffentlichkeit abgehalten, Goethe-Geburtsjahre gefeiert. Kein anderes Werk der deutschsprachigen Literatur weist eine vergleichbare Wirkungsgeschichte wie Faust auf (Kammler 2015). Es wird für seine Einbindung im Deutschunterricht plädiert. So war Faust 2015 als ein Abiturthema ausgewiesen. Entsprechend umfangreich war das Angebot an didaktischen Orientierungshilfen und das Publikationsangebot ließe sich als oszillierend bezeichnen. Es lässt sich aber auch eine gegenläufige Tendenz beobachten, deren Vertreter für den Ausschluss von Goethe'schen Werken aus dem ,schulischen Kanon' und somit aus dem Deutschunterricht plädieren (Taube 2010). Im Folgenden soll es um den gegenwärtigen Deutschunterricht gehen. Der Beitrag gliedert sich in drei Teile: zunächst erfolgt ein kurzer historischer Exkurs, anschließend werden Neubearbeitungen von Faust in der Diversität ihrer Erscheinungsformen vorgestellt und schließlich einige didaktisch-methodische Vorschläge für den Umgang mit Faust in dem kompetenzorientierten Deutschunterricht unterbreitet.

\section{GOETHE-REZEPTION: EIN HISTORISCHER EXKURS}

Im 19. Jahrhundert steht Goethe-Rezeption unter dem Anspruch, eine umfassende Bildung zu vermitteln. Biografische Fakten und Kontexte seiner Werke stellen zentrale Bestandteile des Deutschunterrichts dar und sind entsprechend der vorherrschenden Methode des literarischen Positivismus für das Verstehen des Gelesenen ausschlaggebend. Die eigentliche Textkenntnis ist zunächst zweitrangig, wenn nicht sogar redundant. Das Faktenwissen über den Autor hilft von ihm Besitz zu ergreifen (Rothe 1897: 19), schafft intime Vertrautheit und ermöglicht auch den gesellschaftlichen Klassen, die über kein Interesse an Literatur verfügen, an dem Klassiker und seiner Verehrung teilzuhaben und die sogenannte Inthronisierung voran $\mathrm{zu}$ führen (Korte 2002). Durch die Verfügbarkeit der vielen biografischen Daten erlangt Goethe seine herausragende Stellung, die sich bis zum Personenkult steigert. Kommt es doch zur Lektüre von Werken, so übernimmt die Lehr- 
person im Einklang mit dem pädagogischen Verhältnis von Chladenius Funktion des „Aufklärers der dunklen Stellen“ (Gadamer 1990: 186). Das Deutungsmonopol liegt bei ihm legitimiert durch den seinem Berufsbild per definitionem inhärenten Wissensvorsprung.

Weitere Aspekte, die mit Goethe'schen Werken in Verbindung gebracht werden, lassen sich in Abhängigkeit von dem jeweiligen Schultyp in zwei sich gegenseitig ausschließende Richtungen aufteilen - Volksverbundenheit einerseits und das humanistische Bildungsideal auf der anderen Seite. Die zunächst genannte Tendenz hat eine besondere Signifikanz für die Volksschulen. Hier werden sie vor dem Hintergrund des folkloren Tons - als Ausdruck des Denkens und des Fühlens des Volkes, als sein Eigentum (Reber 1899 zit. nach Popp 2005: 51) rezipiert. Der Schriftsteller selbst ist ein Übersetzter eines bereits vorhandenen, universalen Diskurses und ein Sprachkanal des Volks (Kittler 1995). Dabei werden die Synergieeffekte sowohl für den Dichter als auch für das Volk bestimmt. Er soll aus den lyrischen Ressourcen - aus der wahren Poesie - geschöpft haben (Rothe 1897: 19), das Volk wiederum wurde mittels Goethes Werke gebildet, denn sie gelten als seiner geistigen Entwicklung angemessen. Bei den Kindern wecken sie lebendigsten Widerhall (Lomberg 1989: 4). Ferner korrespondiert eine solche Einstellung mit dem Bildungsauftrag der Volksschule, der in der Rückbesinnung auf das Volkstümliche, in der Bildung mit patriotischen Zügen und in dem Bestreben, das Individuum in die Volksgemeinschaft zu integrieren und "fürs Leben auszubilden" (ebenda) besteht. Methodisch ist die Rezeption der Gedichte nicht vom rational-analytischem Durchdringen von Form und Inhalt geprägt, als vielmehr eine Art des intuitiven Erfassens darstellt.

In Gymnasien dagegen wird die Volkstümlichkeit mit einer antiintellektuellen Haltung und mit einer bewussten Ablehnung des systematischen, analytischen Denkens sowie einer Beschränkung von Wissen assoziiert. Unter der Prämisse Bildung durch Dichtung zielen die Bemühungen auf die Entfaltung aller im Menschen angelegten Fähigkeiten unabhängig von der praktischen Anwendbarkeit der Lektüre. Erziehung durch Dichtung und Erziehung zur Dichtung, Einführung in die Welt der Schönheit und der Harmonie, ästhetische Erziehung und das humanistische Bildungsideal sind die Schlagwörter. Diese Ausrichtung bedingt auch die Wahl der Werke: Iphigenie nicht Götz, Tasso und Egmont stehen auf dem Lesecurriculum. Dass deutsche Werke im Allgemeinen und die von Goethe trotz der Dominanz des Griechischen und des Lateinischen den Eingang in das Gymnasium finden, ist der Verdienst von Friedrich Niethammer (allerdings gewinnt sein Kanon keine Bedeutung für die schulische Wirklichkeit (Fuhrmann 1993)), von Philipp Raumer und der von Robert Heinrich Hiecke. Der zuletzt erwähnte entwirft ein Unterrichtsmodell und prägt dadurch nicht nur den 
Deutschunterricht, dem in der Periode zwischen Vormärz und der Reichsgründung eine zentrale Rolle als Bildungs- und Gesinnungsfach zukam, sondern die Neue Deutsche Literaturwissenschaft. Beide orientieren sich an sein Werk Interpretationsstunden der deutschen Klassiker. Die methodische Umsetzung basiert auf der den Text zergliedernden Textanalyse. Intendiert werden dadurch die Schulung des logischen Denkens und primär der Aufbau der literarischen Handlungskompetenz.

Mit der Reichsgründung und dem Anbruch des Zeitalters der Industrialisierung vollzieht sich erneut ein Paradigmenwechsel vom humanistischen Bildungsideal hin zum realistischen. Goethes Werke bleiben ein Bestandteil des Unterrichts, allerdings unter einer anderen Rhetorik - ihm und seinem Schaffen wird die nationalerzieherische Wirksamkeit zugeschrieben. Goethe, obwohl er sich zeit seines Lebens gegen die Politisierung wehrte, wird in diesem Kontext als der nationale Dichter vereinnahmt. Seine Werke gelten nun als „nationaler Schatz" und als „Pflicht der Nation“ (Bachmann 1895: 20). Die Formulierungen, dass Goethe sich immer wieder aufs Neue mit Stoffen aus Deutschlands Gegenwart und Vergangenheit auseinandergesetzte, und sie stets die Basis seiner Werke bildeten, ebenso wie die Protagonisten seiner Werke stets die Eigentümlichkeit germanischen Wesens trugen, sind für den damaligen Diskurs kennzeichnend (Lorenz 1999: 6). Dies legitimiert die schulische Lektüre innerhalb der staatlichen Vorgaben und hat zur Folge, dass Faust zum Nationalgedicht des Deutschen avanciert, Dorothea und Hermann und Götz von Berlichingen den Einzug auch in den gymnasialen Literaturunterricht finden. Das gesamte Bildungswesen wird verstärkt für Staatsinteressen nutzbar gemacht. Literatur wird zur Vermittlung geschichtlicher Ereignisse funktionalisiert, die literarische Handlungskompetenz wird nicht länger als ein Ziel des Unterrichts formuliert. Die Kenntnis der Werke - Zitatenwissen - wird zum Bildungsgut. Dies lässt sich weiter verfolgen. In der NS-Zeit wird Faust für proletarische Diktaturen vereinnahmt. So gilt Faust als faschistischer Held, später und im östlichen Teil Deutschlands als Vorreiter des real existierenden und anzustrebenden Sozialismus und Kommunismus und der stalinistischen Propaganda (insbesondere der zweite Teil). In den 1950er-Jahren sind Goethes Werke nach wie vor ein unabdingbarer Bestandteil des Deutschunterrichts. Faust gilt als Nationalbibel, ihr Protagonist verkörpert die deutsche Seele, ist ihr Inbegriff, der Repräsentant des deutschen Nationalcharakters, die Leitfigur und das sittliche Vorbild für Jugend. Allerdings ist es nicht seine moralische Integrität (Gaier 2012), sondern seine Lebensgeschichte, die als Paradebeispiel einer gelungener Persönlichkeitsentwicklung gelesen wird, seine Standfestigkeit gegenüber allen Versuchen von Mephisto, ihn in die Niederungen materiellen Begehrens herabzustürzen, sein Streben nach Höherem, Ideellem, seine Forderung 
nach ständiger Innovation, seine Utopie, paradiesisches Land zu schaffen. Der Aspekt der ,Überzeitlichkeit' und der kanonische Status der Tragödie spielt ebenfalls eine Rolle als Legitimationsgrundlage. Es wird ein Diskurs um Aneignung des literarischen Erbes geführt. Die werkfernen Faktoren - wie Biografie der Autors und der Entstehungskontext treten weitgehend in den Hintergrund, die sogenannte werkimmanente Interpretation behauptet sich als dominierende Unterrichtsmethode. In den inhaltlich-orientierten Lehrplänen sind Goethes Werke und insbesondere Faust ein fester unangefochtener Bestandteil (Thiel 1960 zit. nach Fuhrmann 1993). In den 1970er-Jahren gewinnen Ziele und ihr Erreichen an Signifikanz (Bernsmeier 2015). Die Inhalte sind zunächst zweitrangig und müssen sich diesen gegenüber legitimieren. Faust kann gelesen werden, aber es besteht keine Pflicht, und es brauchen gar nicht Goethes Werke oder Klassiker zu sein. Zwar gibt es Empfehlungen, was im schulischen Unterricht thematisiert werden soll, aber grundsätzlich ist es den Lehrenden überlassen, wie sie die einzelnen Stunden füllen. Eine Ausnahme bildet der Unterricht in den Abiturklassen. Hier sind von Signifikanz die Angaben in Kerncurricula, in den Einheitlichen Prüfungsanforderungen (EPA) sowie Vorgaben für das Abitur von Signifikanz. Die Situation ähnelt sehr der im gegenwärtigen Deutschunterricht vorzufindenden. Der Unterschied besteht darin, dass jetzt nicht Ziele erreicht, sondern Kompetenzen gefördert werden sollen. Man setzt auf die von Schülerinnen und Schülern selbst bestimmte Textbegegnung, auf soziale, affektive und emotionale Zugänge. Vermieden werden sollen die Hinstilisierungen und Deutungen aus der germanistischen Literaturwissenschaft, die den Schülerinnen und Schülern vorgesetzt werden. Es konkurrieren das identifikatorische und das textnahe/ statarische Lesen (Paefgen 1996, 1998), Konzepte die sprachliche und inhaltliche Alterität und Diskrepanzerfahrungen hervorheben, Wahrnehmungs-, Empathieschulung (Menzel 1999) und Vorstellungsbildung, szenische Interpretation (Scheller 2004), Arbeit mit Statuen (Zabka 2015: 15) darstellendes Spiel (Fix 1992). Als Kriterien für die Wahl der im Unterricht zu behandelnden Werke gelten Aktualität, (Epochen)repräsentanz (Exemplarität), Wirkungsmächtigkeit, Länge, Interesse der Schülerinnen und Schüler daran, Vorhandensein von Lektürehinweisen und didaktischen Orientierungsvorschlägen, politische Korrektheit und nicht zuletzt Abiturrelevanz (Fuhrmann 1993, Müller-Michaels 1994, Sieburg 2010, Kammler 2015 und Bernsmeier 2015). Einhergehend mit dem Paradigmenwechsel im Bildungsdiskurs änderten sich die Sinnzuschreibungen und Deutungsansätze. Faust wird nicht mehr als die urdeutschen Charaktereigenschaften und Verhaltensweisen innehabender Protagonist gesehen, sondern vielmehr in seiner Vielschichtigkeit und Ambivalenz. Völker (1999: 193) formuliert die Kontroverse Faust als eine Leitfigur oder ein skrupelloser Verbrecher mit einer ver- 
zweiflungsvollen Ablenkungssucht. Jaeger (2008: 88) bezeichnet Fausts unstillbaren Erkenntnishunger als „Kunst der rastlosen Innovation“, zieht eine Parallele zur Moderne und akzentuiert die destruktive Seite Fausts Ambitionen. Sein Erkenntnisdrang und seine Rastlosigkeit zielen darauf ab, jede Realität zu verschlingen, und dessen Opfer zunächst Margarethe, ihre ganze Familie und dann auch noch Philemon und Baucis sind. Diese Leseart, pointiert formuliert - Verbot des Verweilens als Signatur der Moderne und der damit einhergehende ziellose Geschwindigkeitskult mit dem permanenten Sensationswechsel und mit der Entwertung des momentan Gegenwärtigen - findet sich bei Schmidt 1999, Schlings 1998 und Osten 2003 wieder. Safranski dagegen betont die positiven Aspekte der Hauptfigur als erfahrungs- und erkenntnishungrigen Grenzüberschreiters, indem er vom immanenten Transzendieren spricht (Safranski 2013). Die Kontroverse wird durch Hamacher geschickt durch den Hinweis auf die semantische Offenheit literarischer Texte bzw. „polyseme Semantik“ (2013: 131) gelöst. Weitestgehend konform ist dies mit dem von Korte (2002) vorgeschlagenen Konzept des Deutungskanons. Deutlich wird, dass nach wie Versuche vorgenommen werden, der Titelfigur neue Konturen zu geben, und die Sinnzuschreibung noch nicht abgeschlossen ist.

\section{GOETHE UND FAUST IN DER KINDER- UND JUGENDLITERATUR}

Die literaturwissenschaftliche Forschung zu Faust ist ausufernd, die Literaturdidaktik hat sich mit nahezu gleicher Extension mit diesem Gegenstand beschäftig. Eine weitere Komponente des Faust-Diskurses bilden FaustComics, Mangas, Bilder- und Kinderbücher, Zusammenfassungen Lektürehinweise und Interpretationshilfen, Verfilmungen und Adoptionen. Die Bezugnahmen auf Goethes Werk bzw. generell auf den Fauststoff sind unterschiedlich motiviert. Es kann der Popularität der literarischen Figur geschuldet sein - Ausbeutung des symbolischen Kapitals (Freise 2010) oder auf dem Erfolg des in der Weltliteratur erprobten narrativen Konzepts gründen. Manche von diesen Produktionen stellen einen ernsthaften Versuch dar, Faust in andere Medien zu überführen - Comic von Falk Nordmann (1996) - die anderen lehnen sich lediglich an die wesentlichen Handlungselemente an, verwenden den Text fragmentarisch, parodieren und persiflieren ihn und erzählen schließlich eigenständig weiter. In Kürze seien einige Werke vorgestellt: Es soll mit der Persiflage des Klassikers von Flix (Felix Görmann) begonnen werden, denn sie ist mittlerweile in die didaktischen Orientierungshilfen aufgenommen worden (Radvan, Steiner 2013 und Reinhard-Becker 2015). Er überträgt das Geschehen des klassischen Stoffes 
in die Gegenwart und modernisiert ihn. Faust studiert Medizin, Jura, Philosophie und Theologie und finanziert sein Leben durch die Einnahmen aus seiner Arbeit als Taxifahrer in Berlin-Kreuzberg. Margarethe hat eine türkische Herkunft und stellt ein Gegenteil von Goethes Gretchen dar. Der schwarze Pudel heißt Charlotte von Stein, wurde aber von Faust überfahren. Das führt zu einem Streit zwischen ihm und dem Besitzer des Hundes Wagner. Unverändert ist die für die Handlung ausschlaggebende Wette um Fausts Seele, auch wenn der Himmel nun ein Büro mit multikonfessionellen Mitarbeitern darstellt, und als Wetteinsatz für Fausts Treue zwei Kisten eines alkoholischen Getränks dienen. Aufgrund Gretchens Wanderbiografie verlagert sich der zentrale Konflikt auf das tragische Potenzial einer Liebe zwischen einer Muslimin und einem Deutschen und auf die daraus resultierende Konsequenz des Ehrenmords.

Anders geht Tezuka Osamu vor (s. Literaturhinweise). In seiner MangaInterpretationen Faust und Neo-Faust verbindet er Elemente aus Faust I und Faust II, wenngleich die Szenen aus Faust II enttextualisiert wirken, und die Textnahe nicht besonders groß ist. In der Osamus Version beginnt die Geschichte mit einem Streit zwischen Mephisto und einem Engel, den Mephisto aus dem Paradies auf die Erde wirft. Daraufhin muss sich er vor Gott verantworten. Im Gespräch schlägt der Allmächtige dem Teufel vor, als Kraftprobe die Versuchung seines irdischen Mustergelehrten Faust. Die erste Begegnung zwischen den Beiden erfolgt an einem Osterspaziergang. Der Teufel erscheint in Form eines schwarzen Pudels und wie bei Goethe finden sich die Szenen mit den Ratten und dem magischen Drudenfuß. Es kommen Episoden mit dem Erdgeist und mit dem Verjüngungselixier vor. Anders in das Geschehen eingebunden ist die Begegnung mit Margarete, die eine Prinzessin ist. Nicht einer Orgie gleich, wie bei Goethe ist die Szene mit Walpurgisnacht, auch wenn die ihr beigemessene Bedeutung mit der literarischen Vorlage vergleichbar ist. Mephisto und Faust schauen sich während der Walpurgisnacht ein Theaterstuck Wagner und Homunculus an und erhalten einen Hinweis auf den Verbleib von Helena. Als Faust zum König wird, will er Margarete zurückholen. Sie bevorzugt jedoch den Feuertod. Schließlich erwacht Faust in seinem Studierzimmer und erkennt seinen Wahn. Mephisto will ihn zu sich holen, aber Margarete in der ursprünglichen Gestalt eines Engels rettet letztendlich Fausts Seele. Goethes Text wird von anderen Figuren gesprochen, als dies das Original vorsieht. Stephan Köhn bezeichnet diesen Manga als "ein wildes Potpourri des Originals“ (Köhn 2010: 38). Erklären lässt sich das durch die Handlungsorientiertheit des Story-Manga. Längere Dispute, innere Zweifel, Darstellung psychischer Befindlichkeiten, die das Original bestimmen, finden medienbedingt zum Zeitpunkt der Entstehung des Mangas von Tetzuka Osamu noch keinen Eingang. 
Osamus Adoptionskonzept von Neo-Faust ist nicht auf die Werktreue in Bezug auf Goethes Faust ausgerichtet, sondern beabsichtigt vielmehr durch gezielte inhaltliche wie formale Strategien der Übertreibung und Verfremdung bei Rezipienten Irritation und Distanzierung hervorzurufen. Die Anknüpfungspunkte und Schnittstellen zur Goethes Faustfigur werden konsequent unterlaufen. Der für die Handlung ausschlaggebende verhängnisvolle Pakt wird beibehalten, damit enden jedoch die Berührungspunkte: Die Handlung wird in die 1970er-Jahre verlagert, Mephisto hat eine weibliche Gestalt, Faust selbst - zunächst Professor Ichinoseki nach der Einnahme des Verjüngungstranks verwandelt es sich in Sakane Daiichi - ist Assistent für die Gentechnologie und erst später Professor. Er muss diese Stellung erneut erreichen. Das gelingt ihm indem er seine eigenen Notizen angefertigt als Professor Ichinoseki zur Urknalltheorie entdeckt. Margarete - Takada Mariko - ist zu Beginn der Handlung Studentin der Nagano-Universität. Im weiteren Verlauf befindet sie sich in der Behandlung in einer psychiatrischen Klinik. Erst nach mehr als zehn Jahren, in der Abschlussepisode des Mangas, treffen sie sich wieder. Damit endet Neo-Faust.

Im Bilderbuch Osterspaziergang mit Illustrationen von Klaus Ensikat (2012) wird der Text des Originals ohne Veränderungen übernommen. Der Textauszug entstammt Goethes Faust und somit wird das auf dem Titelblatt gemachte Versprechen „eine Hommage an Goethes weltbekannte Verse aus dem Meisterwerk Faust zum alljährlichen Naturwunder des wiederkehrenden Frühlings" eingehalten.

Felix Huby (1980) nimmt sich als Inspiration auch den Fauststoff, allerdings rekurriert er auf die populäre Schwarzmagiegestalt. Sein Faust strebt nach Wissen und klärt auf. Er beschwört zwar den Teufel, schließt aber mit ihm keinen Pakt ab. Im Gegenteil sucht er diese Erscheinung auf seinen $\mathrm{Zu}$ stand der geistigen und körperlichen Erschöpfung nach mehreren Tagen des Hungerns und der intensiven Arbeit zurückzuführen und verneint dadurch Existenz des Dämons. Überschneidungen finden sich in den Episoden mit dem Wein und mit den Nasen. Anders sind Orte und Kontexte, in die diese eingebunden sind. Es tauchen Namen von Götz von Berlichingen und von Franz von Sickingen auf. Um diese und viele andere Bezugnahmen zu erkennen, bedarf es jedoch einer intertextuellen Enzyklopädie und des Wissens der beiden Werke.

Faust nach Tragödie von J. W. Goethe aus der Reihe Weltliteratur für Kinder will im eigenen Wortlaut Groß und Klein auf verständliche unterhaltsame Art den Zugang zu klassischen Werken und Themen der Weltliteratur eröffnen. In der Tat wird Faust nacherzählt, auch wenn sehr viele Reduktionen vorgenommen werden. Das Ende wird aus den pädagogischen Gründen Faust II entnommen. Interessant ist auch der Hinweis der Autorin, dass sich 
die Sprache und der Stil der klassischen Vorlage noch unverkennbar wiederspiegeln. Die Originalzitate sind kursiv gesetzt und sollen einen möglichst authentischen ersten Eindruck des Goethes Werks vermitteln (Kindermann 2005).

Rafik Shamis Der geheime Bericht über den Dichter Goethe, der eine Prüfung auf einer arabischen Insel bestand (2001) kann als ein Lob auf den Fürsten der Dichtung gelesen werden. Thomas oder Tuma, wie er genannt wird, stellt der Kommission, deren Aufgabe darin besteht, für die Bibliothek des Sultans die besten Bücher der Welt auszusuchen, Goethes Werke - von Werther, Wilhelm Meister, Reineke Fuchs, Faust, Zauberlehrling über Wahlverwandtschaften, Liebesgedichte und schließlich West-östlichen Divan vor. Nicht unerwähnt bleiben Goethes naturwissenschaftliche Arbeiten über Farblehre und sein publizistischer Angriff auf Isaak Newton. So erhalten die (jungen) Leser einen guten Überblick über das Schaffen des Schriftstellers.

Als Einstieg, als Hinführung zum Werk, zur Erarbeitung seines Grundgerüsts, der zentralen Konflikte und der Beschaffenheit der Charaktere können die Interpretationen des Fauststoffs durchaus zum Gegenstand der unterrichtlichen Betrachtung gemacht werden. Es können Motive und Handlungsweisen der Figuren, Ästhetik der Sprache untersucht und in Ansätzen bewertet werden. Es sollte allerdings nicht dabei belassen werden. Man kann den Blick auf Textstellen lenken, Figuren, Kontexte, in denen diese in die Handlung eingeführt werden und ihre Entwicklung, Orte und Themen notieren und so für eine spätere unterrichtliche Betrachtung und Besprechung greifbar machen. Eine solche Vorgehensweise sollte allerdings die medial bedingten Formen des Erzählens wie die damit verbundenen Text- und Bildverstehensprozesse nicht aus dem Blick verlieren. D.h. es geht nicht darum die visuellen Darstellungen im Fall von Comics oder Kinderbücher als Suchtexte zum Original (Köhn 2010) zu verstehen und diese vor diesem Hintergrund zu rezipieren. (Zum Potenzial der unterrichtlichen Arbeit mit Comics Kuzminykh 2014). Arbeitet man mit Bilderbüchern, so können Text-Bild-Beziehungen zum Gegenstand des unterrichtlichen Gesprächs werden oder aber eigene kreative Zugänge erprobt werden.

\section{GOETHES FAUST IM GEGENWÄRTIGEN DEUTSCHUNTERRICHT}

Comics, Adoptionen oder das Original im kompetenzorientiertem Deutschunterricht - so lautete die eingangs gestellte Frage. Sieburg (2010) aufgreifend die Kritik von Fuhrmann (1993), Gidion (1992) oder Karg (2003) verurteilt den gegenwärtigen kompetenzorientierten Unterricht. Er wandle 
die Komplexität und den Kunstcharakter der Literatur zu einem Übungsmedium beim Erwerb bestimmter Kompetenzen um und würdige die literarischen Werke herab. Es soll an dieser Stelle nicht für die Rückkehr zu Inhalten plädiert und nicht den Fragen, was Literatur ist und was sie leisten muss, nachgegangen werden, sondern ein Vorschlag für einen Literaturunterricht unterbreitet werden, der Texte ausgehend aus ihrer Rezeption und ihrem Verständnis - in diesem Fall Goethes Faust - würdigt, ohne jedoch ihre Inthronisation zu verfolgen.

Eine Voraussetzung für die intendierte unterrichtliche Vorgehenswiese mit Goethes Werk ist die Textkenntnis des Originals. Dabei sollten die Schülerinnen und Schüler gebeten werden, , aktiv` zu lesen. Dies kann mittels der aus dem amerikanischen Sprachraum kommenden Methode "talking to the text" (Masanek 2008), als das textnahe Lesen (Paefgen 1996, 1998) erfolgen, oder aber können die Leseeindrücke und Gedanken, Assoziationen und (erste) Deutungshypothesen in einem Lesetagebuch festgehalten werden (Mikota 2013). Nach der erfolgten unterrichtlichen Arbeit können sie ihre Notizen und neu erworbene Kenntnisse abgleichen, ihre Sinngebungen und Interpretationen revidieren oder ergänzen. Dadurch sehen sie den Text nicht nur anders, denken über das Gelesene und eigene Zugänge nach, sondern gewinnen auch Einblicke in den Textverstehensprozess - der hermeneutischen Zirkel (Gadamer 1993: 57 ff.) oder Spirale (Bolten 1985) und in die Polyvalenz der Texte, die als Literatur gelten (Karg/ Kuzminykh 2014).

Es wäre vom Vorteil, wenn die Schülerinnen und Schüler das Original rezipierten, wenngleich die Vorstellung, eine Lerngruppe von der Auslegungsgeschichte fern zu halten und eine gänzlich unvoreingenommene Lektüre zu garantieren, aufgrund zahlreicher Zusammenfassungen oder Überführungen in ein anderes Medium utopisch ist. Es kann ein gemeinsamer Theaterbesuch geplant werden, um lebendige Wirklichkeit einer Aufführung zu erleben, doch würde dies die Lektüre nicht ersetzen können, vielleicht aber erleichtern, da die Handlung und das Grundgerüst bekannt wären. Auch unterliegt eine Theateraufführung einer Flüchtigkeit und legt die Geschwindigkeit der Rezeption fest. Die Zuschauer haben keine Möglichkeit, wie sie noch beim Medium Film gegeben ist, die Stellen noch einmal anzuschauen und den Vorgang zu verlangsamen. Ferner ist der Unterricht auf reproduzierbares Material angewiesen. Der Vorteil eines Theaterbesuchs, wie jedoch auch jedes intermedialen Vergleichs, würde abgesehen von den bereits erwähnten Vorzügen darin bestehen, dass man die vorgefundene Interpretation thematisieren könnte. Dabei geht es nicht darum, das eine Medium gegen das andere auszuspielen, sondern darum, dass mit den Schülerinnen und Schülern erarbeitet wird, dass jede Überführung einer literarischen Vorlage in ein anderes Mediums eine (neue) Interpretationsleis- 
tung darstellt. Schließlich machen sie auch nichts anderes, wenn sie lesen sie interpretieren und schreiben dem Gelesenen Bedeutungen $\mathrm{zu}$, finden ihre eigenen Deutungspositionen und begründen diese. Es gibt dabei keine richtige bzw. bessere Interpretation und kein richtiges Verstehen, sondern es sind stets eigene Assoziationen und eigene Deutungen - die literarischen Texte oder solche die als Literatur gelten, zeichnen sich gerade durch die Polyvalenz der Bedeutungen: Es ist ihr konstituierendes Merkmal, dass sie sich den eindeutigen Sinnzuschreibungen entziehen. Nimmt man von der Unterscheidung zwischen literarischen und pragmatischen Texten Abstand (Kintsch 1994 und Zwaan 1996) und geht man von der Vorstellung von Literatur als einem Rezeptionsphänomen aus, so gewinnt die vom Rezipienten eingenommene Lesehaltung - literary mode - an Relevanz (Karg/ Kuzminykh 2014). Dieser Lesemodus zeichnet sich durch die Bereitschaft zu einer höheren Ambiguitätstoleranz. Bereits in den vorschulischen Vorlesesituationen entwickeln Kinder literarische Rezeptionsmuster. Die (polyvalenten) Bedeutungskonstruktionen erflogen vor dem Hintergrund der vorhandenen Wissensbestände. Es ist daher nur logisch, dass je ausdifferenzierter und vielschichtiger das Wissen ist, desto mehr liest man aus dem Text heraus, und man versteht ihn auch anders. Auch das sollte zu Thema des Unterrichts werden.

Gewinnbringend ist an dieser Stelle die Arbeit mit Stofftradition, die Goethe in seine Faust-Version miteinfließen ließ. In diesem Zusammenhang kann sich das Konzept der Intertextualität zum Thema der unterrichtlichen Arbeit gemacht werden. Die Intention besteht dabei darin die diversen Akzentuierungen zu erkunden. Als Prätexte können das Buch Hiob, Historia $z u$ Dr. Johann Fausten (1587), Lebensdarstellung von Georg Faust (1480-1540 oder 1541), Tragical History of the Life and Death Doctor Faustus von Christopher Marlowe, Puppenspiel in Wandertheatern nach Marlowes Motiv, Lessings Faust im 17 Literaturbrief, Prozesse der Kindermörderinnen, Shakespeares Sommernachtstraum und als Nachtexte beispielsweise Thomas Manns Doktor Faustus und Puškins Dämon herangezogen werden. Die Erkenntnis, die man dabei gewinnt, ist dass die Literatur nationale Grenzen überwindet und Faust ein Bestandteil der Weltliteratur ist. Die Schüler daran teilhaben $\mathrm{zu}$ lassen ist als eine genuine Aufgabe des Literaturunterrichts. Methodisch ließe sich das mittels Poster, als Gallery-Walk/ Gallery-Table bzw. mit der Methode Welt-Café umsetzten (Mattes 2011). Geeignet wären Kurzvorträge und Präsentation mit dem Medieneinsatz als ein authentischer Anlass zur Förderung der Medienkompetenz.

Ganz im Sinne des handlungsorientierten Deutschunterrichts wären die Schülerinnen und Schüler im Anschluss an die Lektüre nach Themen zu befragen, die sie gerne besprechen und nach Textpassagen, die sie einer ge- 
nauen Betrachtung unterziehen würden. Man könnte dies auf Postern oder Kärtchen festhalten lassen. Somit stünde das zur Verfügung und könnte jederzeit aufgegriffen werden. man könnte sich auch auf die Arbeitsweisen einigen. Die Schüler wären dann in die Gestaltung des Unterrichts aktiv miteinbezogen. Die Lehrperson kann auch ihre Vorschläge einbringen und begründen, darf sie jedoch nicht aufzwingen. Denkbar wären folgende Schwerpunkte: Debatte über die Natur der Menschen und Menschenbilder (Mephisto und der Herr) - anthropologische Leseart Faust (Gaeir 2012) Faust als Menschheitsdrama, Wertetafeln der jeweiligen Protagonisten und dementsprechend Figurencharakteristiken - eigene und fremde - und vollzogene Entwicklung im Laufe der Handlung, Machtverhältnis und Machtverteilung, Bedingungen der Wette, Fausts Ambivalenz, Zerrissenheit, Dialektik der Selbstverwirklichung in diversen Lebensbereichen - in der Wissenschaft, in der Liebe, in der Gesellschaft und Politik und im Leben, Lebensdrang und Erkenntnishunger sowie Verzweiflung an der Unmöglichkeit diese zu befriedigen, Ambitionen und Enttäuschungen, Existenzkrise und Ausbruchsversuche, Transfer auf die modernen Gesellschaften, historische Kontexte und Epochenrepräsentanz bzw. Suche nach den Spuren von Epochen und Richtungen (Mittelalter, Klassik, Aufklärung, Nihilismus, Sturm und Drang, Neuzeit), der unikale Aufbau der Tragödie, literaturwissenschaftliche Aspekte (Reimstruktur, Sprache, stilistische Mittel), volkstümliche Elemente und die bereits erwähnten intertextuellen Bezüge, antichristliche und antireligiöse Motive und Fausts Vorstellung vom Gott, Popularität und Faszination des Fauststoffs und Gründe hierfür sowie Kanonisierung des Textes. Ein Thema könnte auch die Erkundung des Diskurses um Faust sein - politische Vereinnahmung und Zensur.

Der Einstieg in die Sequenz könnte auch über Auszüge aus Texten mit Faustmotiv, Bilder oder über Zitate aus Goethes Faust erfolgen. Das würde den Blick auf die jeweiligen Textstellen oder Figuren lenken, die im Sinne einer komparativen Arbeit besprochen werden können, und bei Schülerinnen und Schüler einen Wiedererkennungseffekt und Motivation hervorrufen. Denkbar ist die Einbeziehung des Konzeptes der szenischen Interpretation (Scheller 2004) oder das Erstellen von Standbildern bzw. Statuen (Zabka 2015) mit der Rezitation ausgewählter Passagen, das poetische, expressive, klärende oder rhetorische Schreiben (Abraham 2012). In Betracht sollten Projektmethode und freie Arbeitsphasen, kreative Methoden und Facharbeiten gezogen werden - all das, was Literatur möglichst vielseitig kennenlernen und erfahren lässt. Dabei sollte es bei den aufgezählten unterrichtlichen Vorgehensweisen nicht um die Vorstellung gehen, dass der Text besser verstanden wird, wenn die Schülerinnen und Schüler ihn laut vorlesen oder in den jeweiligen Rollen sprechen, oder etwas anderes mit ihm tun, sondern 
um das Erarbeiten des Textverstehens und um sein ,Verhandeln' sowie darum, sie zum Nachdenken über das Gelesene und das Verstandene zu bewegen und Recherchearbeit zum Faust zu initiieren und somit Wissen aneignen zu lassen. Der Text gibt Hinweise, reagiert auf das gesellschaftliche Leben, reflektiert es, kritisiert, stellt in Frage, akzentuiert, ironisiert und greift diverse Facetten und den historischen Kontext der deutschen Gesellschaft, Werte und Normen, (kulturelle) Befindlichkeiten und schließlich den kulturellen Prozess bzw. den kulturellen Habitus auf. Das zu erkennen und zu deuten, ist die Leistung der Schülerinnen und Schüler und die Aufgabe des Unterrichts, sie dazu zu befähigen.

Es sind einige Vorschläge für die gemeinsame und für die individuelle Arbeit mit dem Text, mit Figuren, Textstellen und Themen, mit intertextuellen Bezugnahmen und mit zeitlichen Kontexten gemacht worden, jedoch es ist nur eine kurze Skizze und keinesfalls eine erschöpfende Darstellung, wie mit dem Text verfahren werden kann. Die methodische Diversität ist für die Deutungsarbeit vom Vorteil. Sie garantiert zum einen Wissenserwerb und Verstehen der literarischen Vorlage, zum anderen verhelfen die vielseitigen Zugänge den Schülerinnen und Schülern zur Erkenntnis, dass kein (literarischer) Text rein individuelle Kreation ist und für sich steht, sondern mit den Worten von Kirtsten Adamzik in ein „Universum von Texten“ (Adamzik 2004: 255) eingebettet ist, sich auf Prätexte bezieht und Nachtexte hervorruft. Faust ist das beste Beispiel dafür.

\section{LITERATURVERZEICHNIS}

Abraham, U. 2012. Filme im Deutschunterricht. Seelze-Velber: Kallmeyer/ Klett.

Adamzik, K. 2004. Sprache: Wege zum Verstehen. Tübingen: Francke.

Bachmann, F.1895. Wolfgang von Goethe. Gedichte. (Auswahl) Für den Schulgebrauch herausgegeben. Leipzig Freytags Schulausgaben klassischer Werke für den deutschen Unterricht.

Bernsmeier, H. 2015. Kanonisierungseffekte und Klassikerlektüre/ schriftliche Abiturprüfungen/ Bildungsstandards. In: Karg, I. / Jessen, B. (Hrsg.). Kanon und Literaturgeschichte. Frankfurt a.M.: Peter Lang, 129-145.

Bolten, J. 1985. Die Hermeneutische Spirale: Überlegungen zu einer integrativen Literaturtheorie. In: Poetica. Zeitschrift für Sprach- und Literaturwissenschaften, 3/ 4, 355-371.

Damrosch, D. 2003. What is world literature? New Jersey: Princeton University Press.

Hamacher, B. 2013. Einführung in das Werk Johann Wolfgang von Goethes. Darmstadt: Wissenschaftliche Buchgesellschaft.

Fix, M. 1992. „... Und unten zerschellt das Gerippe.“ Inszenierung der Ballade Totentanz von J. W. Goethe. In: Praxis Deutsch, 115, 46-51.

Freise, M. 2010. Die inneren Werte der Literatur und der große Unterschied. In: Freise, M. / Stockinger, C. (Hrsg.). Wertung und Kanon. Heidelberg: Winter, 13-29.

Fuhrmann, H. 1993. Die Furie des Verschwindens: Literaturunterricht und Literaturtradition. Würzburg: Königshausen und Neumann. 
Gadamer, H.-G. 1990. Hermeneutik I. Wahrheit und Methode. Grundzüge einer philosophischen Hermeneutik. In: Gesammelte Werke in zehn Bänden. Bd. I. Tübingen: Mohr.

Gadamer, H.-G. 1993. Hermeneutik II. Wahrheit und Methode. Ergänzungen. Register. In: Gesammelte Werke in zehn Bänden. Bd. II. Tübingen: Mohr.

Gaier, U. 2012. Lesearten zu Goethes Faust. Eggingen: Edition Isele.

Gidion, J. 1992. „Sie werden lachen - Goethe“ Bemerkungen zu den Chancen eines Klassikers in der Schule. In: Neue Sammlung, 2, 215-232.

Jaeger, M. 2008. Global Player Faust oder das Verschwinden der Gegenwart. Zur Aktualität Goethes. Berlin: wjs Verlag.

Kammler, C. 2015. Goethes Faust. In: Praxis Deutsch, 250, 4-12.

Karg, I. 2003. Deutschunterricht für Europa. Frankfurt a.M.: Peter Lang.

Karg, I. / Kuzminykh, K. 2014. Sprache und Literatur als Bildungskomponenten. Diskurs, Historie und Empirie. Frankfurt a.M.: Peter Lang.

Kintsch, W. 1994. Kognitionspsychologische Modelle des Textverstehens: Literarische Texte. In: Reusser, K. / Reusser-Weyeneth, M. (Hrsg.). Verstehen. Psychologischer Prozess und didaktische Aufgabe. Bern: Huber, 39-55.

Kittler, F.1995. Aufschreibsysteme 1800-1900. München: Fink.

Korte, H. 2002. K wie Kanon und Kultur. Kleines Kanonglossar in 25 Stichwörtern. In: Arnold, H. L. (Hrsg.). Text+Kritik. Sonderband. München: Richard Boorberg.

Köhn, S. 2010. Deutsche Klassik einmal anders. Oder Wie Goethes Faust interessante Einblicke in den japanischen Comic gewähren kann. In: Frederking, V. / Hartmut, J. / Josting, P. (Hrsg.). Medien im Deutschunterricht. Jahrbuch 2009. München: kopaed.

Kuzminykh, K. 2014. Comics und graphic novels im multikulturellen Deutschunterricht. In: Glottodidactica - An International Journal of Applied Lingiustics, 41, 2, 75-87.

Link, J. / Link-Heer, U. 1980. Literatursoziologisches Propädeutikum. München: Fink.

Lomberg, A. 1899. Präparationen zu deutschen Gedichten. Nach Herbartischen Grundsätzen ausgearbeitet. Zweites Heft: Goethe und Schiller. Langensalza: Hermann Bayer \& Söhne.

Lorenz, H. 1999. „Erziehung würde Tyrannei sein, wenn sie nicht zur Freiheit führte!“ Über Johann Friedrich Herbarts (1776-1841) Leben und Werk aus neuer Sicht. Oldenburg: Wissenschaftliche Buchgesellschaft.

Masanek, N. 2008. Talking to the Text. Schreibend Texte verstehen. In: Praxis Deutsch, 210, 22-25.

Mattes, W. 2011. Methoden für den Unterricht. Paderborn: Schöningh.

Menzel, W. 1999. Der Rhythmus der Verse: Goethes Meeres Stille und Glückliche Fahrt. In: Praxis Deutsch, 26, 39-47.

Mikota, J. 2013. Literarisches Lernen mit Lesetagebüchern. Carlsen in der Schule. Ideen für den Unterricht. Methoden für Deutschunterricht und Leseförderung. Thema des Monats: Februar 2013 Literarisches Lernen mit Lesetagebüchern. Hamburg: Carlsen <https://www.carlsen.de/sites /de fault/files/1302_Metamodell_Lesetagebuch.pdf> [aufgerufen am: 12.07.2015].

Müller-Michels, H. 1994. Was bleibt? Begründung eines Kanons der Denkbilder. In: Deutschunterricht, 1, 2-10.

Osten, M. 2003. „Alles velizoferisch“ oder Goethes Entdeckung der Langsamkeit. Zur Modernität eines Klassikers im 21. Jahrhundert. Frankfurt a. M.: Peter Lang.

Paefgen, E. K. 1996. Schreiben und Lesen. ästhetisches Arbeiten und literarisches Lernen. Opladen: Westdeutscher Verlag.

Paefgen, E. K. 1998. Textnahes Lesen. 6 Thesen aus didaktischer Perspektive. In. Belgrad, J. / Fingerhut, K. (Hrsg.). Textnahes Lesen. Annäherungen an Literatur im Unterricht. Baltmannsweiler: Schneider Hohengehren, 14-23. 
Popp, K. 2005. Vorbild oder Denkbild? Goetherezeption im Deutschunterricht des späten 19. Jahrhunderts und im aktuellen Literaturunterricht. Frankfurt a. M.: Peter Lang.

Radvan, F. / Steiner, A. 2013. Faust. Der Tragödie erster Teil. Berlin: Cornelsen.

Reinhardt-Becker, Elke. 2015. Wege zu Faust. Eine Unterrichtsreihe zur Graphic Novel Faust. Der Tragödie erster Teil von Flix. In: Praxis Deutsch, 250, 12-19.

Rothe, B. 1897. Erläuterungen zu Goethes Werken für Schulgebrauch und Selbststudium als literaturkundliches Repetitorium. Breslau: Hirt.

Safranski, R. 2013. Goethe. Kunstwerk des Lebens. Biografie. München: Hanser.

Scheller, I. 2004. Szenische Interpretation. Seelze: Klett / Kallmeyer.

Schlings, H.-J. 1998. Faust Verzweiflung. In: Goethe-Jahrbuch, 115, 97-123.

Schmidt, J. 1999. Goethes Faust. Erster und Zweiter Teil - Grundlagen - Werk - Wirkung. München: Beck.

Sieburg, M. 2010. „Wozu Klassiker in der Schule?" Zwei Statements im Rahmen der Podiumsdiskussion zur Vortragsreihe. In: Freise, M. / Stockinger, C. (Hrsg.). Wertung und Kanon. Heidelberg: Winter, 139-145.

Taube, Viola 2010. Klassiker in der Schule. <https://www.youtube.com/watch?v=T6xIDHB83IU> [abgerufen am: 12.07.2015].

Völker, K. 1999. Faust ein deutscher Mann. Die Geburt einer Legende und ihr Fortleben in den Köpfen. Veränderte und erweiterte Neuausgabe. Berlin: Wagenbach.

Zabka, T. 2015. Fausts Paradies - die Hölle. Szenische Interpretation und Wertung der Sozialutopie. In. Praxis Deutsch, 250, 36-43.

Zwaan, R.1996. Toward a Model of Literary Comprehension. In: Britton, B. / Graesser, A. (Hrsg.). Models of understanding Text. Mahwah NJ: Lorenz Erlbaum Associates, 241-257.

\section{KINDERBÜCHER UND COMICS IN AUSWAHL}

Bedürftig, F. / Kirsch, C. 2001. Goethe. Die Comic-Biographie. Berlin: Ehapa Comic Collection.

Goethe, J. W. / Ensikat 2012. Osterspaziergang mit Illustrationen von Klaus Ensikat. Berlin: Kindermann Verlag.

Huby, F. 1980. Das abenteuerliche Leben des Doktor Faust. Stuttgart: Thienemann.

Flix (Görmann, F.) 1998. Who the fuck is Faust? Eine Comic-Tragödie in 7 Tagen. Berlin: Carlsen.

Kindermann, B. 1995 und 2005. Faust. Nach Johann Wolfgang von Goethe mit Bildern von Christiane Mitzkus. 5. Auflage. Berlin: Kindermann Verlag.

Nordmann, F. 1996. Faust der Tragödie erster Teil. Hamburg: B\&K.

Osamu, T.o J. Faust und Neo-Faust. <http://tezukaosamu.net/en/manga/418.html> [abgerufen am: 12.07.2015].

Schami, R. / Gutzschhann, U.-M. 2001. Der geheime Bericht über den Dichter Goethe, der eine Prüfung auf einer arabischen Insel bestand. München: $\mathrm{dtv}$.

Seehafer, K.2008. Goethe für die Schule. Zusammenfassungen du Lektürehilfen der wichtigsten Werke. Berlin: Arena. 
